\title{
UNIMODULAR INVARIANTS OF TOTALLY REAL TORI IN $\mathbb{C}^{n}$
}

\author{
XIANGHONG GONG
}

\begin{abstract}
We study the global invariants of real analytic manifolds in the complex space with respect to the group of holomorphic unimodular transformations. We consider only totally real manifolds which admits a certain fibration over the circle. We find a complete set of invariants for totally real tori in $\mathbb{C}^{n}$ which are close to the standard torus. The invariants are obtained by an analogous classification of complex-valued analytic $n$-forms on the standard torus. We also study the realization of certain exact complexvalued analytic $n$-forms on the standard torus through non-critical totally real embeddings.
\end{abstract}

\section{INTRODUCTION}

In this paper we study unimodular invariants of an immersed or an embedded $n$-dimensional real manifold $M$ in complex space $\mathbb{C}^{n}$ with respect to the holomorphic $n$-form $\Omega=d z_{1} \wedge \ldots \wedge d z_{n}$. We shall consider an immersion or an embedding $\varphi: M \rightarrow \mathbb{C}^{n}$ which is totally real, i. e.

$$
\omega_{\varphi}=\varphi^{*} \Omega \neq 0 .
$$

The complex $n$-form $\omega_{\varphi}$ is decomposed into

$$
\omega_{\varphi}=e^{i \mu} \nu, \quad i=\sqrt{-1},
$$

in which $\mu(\bmod 2 \pi)$ is a real function and $\nu$ is a real $n$-form. The decomposition (1.2) is uniquely determined by the requirement that $\nu$ is either a volume form when $M$ is an oriented manifold, or a positive $n$-form of odd kind when $M$ is non-orientable. We say that the totally real immersion $\varphi: M \rightarrow \mathbb{C}^{n}$ is non-critical, if

$$
\tau=e^{i \mu}: M \rightarrow S^{1}
$$

is a submersion.

1991 Mathematics Subject Classification. Primary 32F25.

Key words and phrases. normal form, totally real torus, non-critical immersion.

Partially supported by NSF grant DMS-9304580 at the Institute for Advanced Study. 
There has been extensive investigations on real submanifolds in $\mathbb{C}^{n}$, especially since the work of E. Bishop [2]. In association with the complex tangents, the topology of an immersed submanifold in complex space has been studied by H. F. Lai [10], S. M. Webster [17], [18], and most recently, by F. Forstnerič [5]. In [20], R. O. Wells proved that if an orientable compact manifold $M$ admits a totally real embedding in $\mathbb{C}^{n}$, then its Euler number $\chi(M)$ and Pontrjagin classes must vanish.

From the definition of the non-critical totally real immersion, we have the following.

Proposition 1.1. Let $M$ be a connected compact smooth manifold of dimension $n$. If $M$ admits a non-critical totally real immersion in $\mathbb{C}^{n}$, then its fundamental group $\pi_{1}(M)$ is infinite and the Euler number $\chi(M)$ vanishes.

As a consequence, we obtain

Corollary 1.2. The sphere $S^{n}$ has a non-critical totally real immersion in $\mathbb{C}^{n}$ if and only if $n=1$. If a compact surface $M$ admits a non-critical totally real immersion in $\mathbb{C}^{2}$, then $M$ is either a torus or a Klein bottle.

We mention that the totally real embedding of the Klein bottle in $\mathbb{C}^{2}$ given by W. Rudin [13 is indeed non-critical. In [21, p. 26], A. Weinstein constructed a Lagrangian (whence totally real) immersion of $S^{n}$ into $\mathbb{C}^{n}$ for all $n$. An explicit totally real embedding of $S^{3}$ in $\mathbb{C}^{3}$ was given by P. Ahern and W. Rudin [1]. In [5], Forstnerič proved that all orientable compact surfaces admit totally real immersions in $\mathbb{C}^{2}$, and that a non-orientable surface has a totally real immersion in $\mathbb{C}^{2}$ if and only if its genus is even.

Let $M_{1}, M_{2}$ be two totally real and real analytic compact submanifolds of $\mathbb{C}^{n}$ with dimension $n$. From the Weierstrass approximation theorem, one knows that $M_{1}$ is equivalent to $M_{2}$ through biholomorphic mappings defined near $M_{1}$ whenever $M_{1}$ and $M_{2}$ are diffeomorphic by smooth mappings. In this paper, we shall study when $M_{1}$ is equivalent to $M_{2}$ by a unimodular transformation, i. e. by a biholomorphic transformation $\Phi$ defined near $M_{1}$, which satisfies the relation $\Phi^{*} \Omega=\Omega$. In [6], we proved that locally, a non-critical totally real $n$-dimensional analytic submanifold $M$ in $\mathbb{C}^{n}$ has only one unimodular invariant when $n \geq 2$. Globally, we shall see that one of unimodular invariants of $M$ is the total volume

$$
\rho_{0}=\int_{M} \nu
$$


Namely, two real-valued analytic volume elements $\nu_{1}, \nu_{2}$ on a compact manifold $M$ are equivalent by an analytic diffeomorphism of $M$ if and only if they have the same volume, a result due to J. K. Moser [11].

The main results of this paper concern the unimodular invariants of tori in $\mathbb{C}^{n}$ which are perturbations of the standard torus

$$
T^{n}:\left|z_{j}\right|=1, \quad 1 \leq j \leq n .
$$

Let $A_{r} \subset \mathbb{C}^{n}$ be the annulus defined by

$$
e^{-r}<\left|z_{j}\right|<e^{r}, \quad 1 \leq j \leq n
$$

For a holomorphic mapping $f=\left(f_{1}, \ldots, f_{n}\right): A_{r} \rightarrow \mathbb{C}^{n}$, we denote

$$
\|f\|_{r}=\sup \left\{\left|f_{j}(z)\right| ; z \in A_{r}, 1 \leq j \leq n\right\} .
$$

We have the following result.

Theorem 1.3. Let $M$ be an embedding of $T^{n}$ in $\mathbb{C}^{n}$ given by a mapping $\varphi$ which is holomorphic in $A_{r_{0}}$. Then there exists a positive number $\epsilon_{0}$, which is independent of $r_{0}$, such that for $n \geq 2,0<r_{0}<1$, and

$$
\|\varphi-\operatorname{Id}\|_{r_{0}} \leq \epsilon_{0} r_{0}^{4}
$$

$M$ is unimodularly equivalent to an embedding of $T^{n}$ defined by a mapping

$$
\psi:\left(z_{1}, z^{\prime}\right) \rightarrow\left(\zeta^{-1} g\left(\zeta z_{1}\right), z^{\prime}\right)
$$

with $z^{\prime}=\left(z_{2}, \ldots, z_{n}\right)$ and $\zeta=z_{2} \ldots z_{n}$, where $g$ is given by

$$
\frac{d}{d \theta_{1}} g\left(e^{i \theta_{1}}\right)=\rho_{0} e^{i\left(\theta_{1}+k\left(\theta_{1}\right)\right)}
$$

for a $2 \pi$-periodic real function $k$ satisfying $\int_{0}^{2 \pi} k\left(\theta_{1}\right) d \theta_{1}=0$. Furthermore, $k\left(\theta_{1}\right)$ is uniquely determined by $M$ up to a translation $\theta_{1} \rightarrow \theta_{1}+\pi$.

We now consider an immersion problem as follows. Given a fibering $\tau$ in the form (1.3) and a real $n$-form $\nu$ on $M$, we ask whether there exists a non-critical totally real immersion $\varphi: M \rightarrow \mathbb{C}^{n}$ such that $\varphi^{*} \Omega=\tau \nu$. A necessary condition is that for the complex-valued $n$-form $\omega=\tau \nu$,

$$
\int_{M} \omega=0
$$

For $M=T^{n}$, we have the following.

Theorem 1.4. Let $\omega=(1+a(z)) \Omega$ be a complex-valued $n$-form on $T^{n}$. Assume that $a(z)$ is holomorphic in $A_{r_{0}}$ and $\omega$ satisfies (1.5). Then there exists $\epsilon>0$ such that for $0<r_{0}<1$ and $\|a\|_{r_{0}} \leq \epsilon r_{0}$, there is a totally real and non-critical analytic embedding $\varphi: T^{n} \rightarrow \mathbb{C}^{n}$ with $\omega=\omega_{\varphi}$ 
We organize the paper as follows. In section 2, we shall discuss fiberings of a compact manifold $M$ over the circle. In particular, we shall use the triviality of fibering $T^{2}$ over $S^{1}$ to show that when $M$ is the 2-dimensional torus, the analytic function $\mu$ in (1.3) has no analytic invariant with respect the whole group of analytic diffeomorphisms of $T^{2}$, except for the number of connected components of the fibers of $\tau$. We then normalize the function $\mu$ on $T^{n}$ by volume-preserving analytic transformations. This normalization, proved later in section 4, is essential for the proof of Theorem 1.3. We shall finish section 2 with a regular homotopy classification of non-critical immersions of $S^{1}$ into the complex plane. In section 3, we shall first discuss the invariants of analytic volume forms, and then give a proof for Theorem 1.3. Section 5 will be devoted to the proof of Theorem 1.4.

\section{INVARIANTS OF NON-CRITICAL IMMERSIONS}

In [16], D. Tischler proved that a fibering $M$ over $S^{1}$ exists whenever $M$ has a smooth foliation given by a closed 1-form. The obstruction theory for the fiberings of $M$ over $S^{1}$ were also studied by F. T. Farrell [4] and others (see the references in [4]). In this section, we shall first discuss a fibering $M$ over $S^{1}$. In particular, We shall see that for an analytic fibering $T^{2}$ over $S^{1}$, the only invariant is the number of connected components of its fiber. Then we shall find a complete set of invariants for perturbations of the trivial fibering $T^{n}$ over $S^{1}$ with respect to volume-preserving bundle maps. Finally, we shall discuss the regular homotopy classification of non-critical immersions of $S^{1}$ in $\mathbb{C}$, which is based on a classical result of H. Whitney and W. C. Graustein [21.

2.1. The fibering $M$ over $S^{1}$. We consider a submersion (1.3). Let $\pi: \mathbb{R} \rightarrow S^{1}$ be the covering mapping with $\pi\left(\theta_{1}\right)=e^{i \theta_{1}}$. With $\pi_{1}\left(S^{1}\right)=$ $\mathbb{Z}$, we put

$$
\tau_{*} \pi_{1}(M)=\mathbb{Z} \cdot d
$$

for some non-negative integer $d$. It is clear that $d \neq 0$; otherwise, $\tau$ has a lifting $\widetilde{\tau}: M \rightarrow R$, which contradicts that $M$ is compact and $\tau$ is a submersion. We now choose a Riemannian metric on $M$. Let $\nabla \mu$ be the gradient of $\mu$ with respect to the Riemannian metric. Since $d \mu \neq 0$ on $M$, then the vector field $\nabla \mu$ vanishes nowhere on $M$. This implies that $\chi(M)=0$, and hence Proposition 1.1 is proved.

It is a classical result of $\mathrm{C}$. Ehresmann [3] that if $M, N$ are connected compact manifolds, and $p: M \rightarrow N$ is a submersion, then $M$ is a fiber bundle over $N$. Arising from the submersion (1.3), the fiber bundle structure on $M$ can be described as follows. Let $\varphi_{t}$ be the flow of $\nabla \mu$. 
On $M \times \mathbb{R}$, consider a well-defined smooth function

$$
F(x, t)=\mu \circ \varphi_{t}(x)-\mu(x) .
$$

One notes that

$$
\frac{d}{d t} F(x, t)=<\nabla \mu\left(\varphi_{t}(x), \nabla \mu\left(\varphi_{t}(x)>\right.\right.
$$

has a positive lower bound. Hence, there is a smooth function $t: M \times$ $\mathbb{R} \rightarrow \mathbb{R}$ such that

$$
F(x, t(x, \alpha))=\alpha .
$$

Now, one defines a smooth family of diffeomorphisms $\psi_{\alpha}$ of $M$ by

$$
\psi_{\alpha}(x)=\varphi_{t(x, \alpha)}(x) .
$$

In fact, one readily sees that $\alpha \rightarrow \psi_{\alpha}$ is a homomorphism, i.e. an $\mathbb{R}$-action on $M$ generated by $\tau$. Now for two points $p, q$ on $S^{1}$ with $q=e^{i \alpha} p$ and $0<\alpha \leq 2 \pi$, we have the Poincaré section mapping

$$
S_{p, q}=\left.\psi_{\alpha}\right|_{\tau^{-1}(p)}: \tau^{-1}(p) \rightarrow \tau^{-1}(q) .
$$

For $F=\tau^{-1}(1)$, we define two local trivializations

$$
\phi_{j}: F \times\left(S^{1} \backslash\left\{(-1)^{j}\right\}\right) \rightarrow \tau^{-1}\left(S^{1} \backslash\left\{(-1)^{j}\right\}\right), \quad j=0,1
$$

with

$$
\phi_{0}(x, p)=S_{1, p}(x), \quad \phi_{1}(x, p)=S_{p,-1} \circ S_{1,-1}(x) .
$$

Therefore, $\tau: M \rightarrow S^{1}$ is a fiber bundle with fiber $F$. Obviously, $\psi_{\alpha}$ is a bundle map between two fiber bundles $\tau: M \rightarrow S^{1}$ and $e^{i \alpha} \tau: M \rightarrow S^{1}$.

By the homotopy sequence of a fibering (see [9], p. 152), we know that $d$ is exactly the number of connected components of fiber $F$. Now the fibering $\tau$ has a factorization $\tau=\left(\tau^{1 / d}\right)^{d}$, where the $d$-th root $\tau^{1 / d}$ is a lifting of $\tau$ for the standard $d$ to 1 covering from $S^{1}$ to itself defined by $z \rightarrow z^{d}$; and $\tau^{1 / d}: M \rightarrow S^{1}$ is a fiber bundle with connected fibers. It is clear that if $\tau^{\prime}, \tau^{\prime \prime}$ are two $d$-th roots of $\tau$, then $\tau^{\prime \prime}=\lambda \tau^{\prime}$ with $\lambda^{d}=1$. Using the $\mathbb{R}$-action on $M$ generated by the fibering $\tau^{\prime}$, one can find a bundle map between the fiberings $\tau^{\prime \prime}$ and $\tau^{\prime}$. Furthermore, let $\tau_{j}: M_{j} \rightarrow S^{1}(j=1,2)$ be two fiberings. Assume that fibers of both $M_{1}$ and $M_{2}$ have $d$ connected components, and that $\tau_{1}^{1 / d}$ and $\tau_{2}^{1 / d}$ are the corresponding $d$-th roots of $\tau_{1}$ and $\tau_{2}$ respectively. Then under bundle maps, $\tau_{1}$ is equivalent to $\tau_{2}$ if and only if $\tau_{1}^{1 / d}$ is equivalent to $\tau_{2}^{1 / d}$. This implies that, for the purpose of the bundle classification, we may only consider a fibering $M \rightarrow S^{1}$ with connected fibers.

We shall introduce some notations. Define a universal covering $R^{n} \rightarrow$ $T^{n}$ by

$$
\pi:\left(\theta_{1}, \ldots, \theta_{n}\right) \rightarrow\left(e^{i \theta_{1}}, \ldots, e^{i \theta_{n}}\right)
$$


For a mapping $\phi: T^{n} \rightarrow T^{m}$, a lifting $\widetilde{\phi}$ of $\phi$ is a mapping from $\mathbb{R}^{n}$ to $\mathbb{R}^{m}$ such that $\pi \circ \widetilde{\phi}=\phi \circ \pi$. Obviously, $\widetilde{\phi}$ can be written as

$$
\theta_{k}^{\prime}=\sum_{l=1}^{n} d_{k, l} \theta_{l}+f_{k}(\theta), \quad d_{k, l} \in \mathbb{Z}, \quad k=1, \ldots, n
$$

for some real functions $f_{k}$ which are $2 \pi$-periodic in each variable $\theta_{j}$. Also, a mapping $\widetilde{\phi}: R^{n} \rightarrow R^{m}$ in the form (2.2) generates a unique mapping $\phi: T^{n} \rightarrow T^{m}$ such that $\widetilde{\phi}$ is a lifting of $\phi$. It is clear that if $\phi$ is a diffeomorphism, so is $\widetilde{\phi}$. Conversely, the degree formula (see [7], p. 189) implies that if $\widetilde{\phi}$ is a diffeomorphism, then $\phi$ is a local diffeomorphism of $T^{n}$ with degree

$$
d=\operatorname{det}\left(d_{k, l}\right) .
$$

In particular, $\phi$ is a diffeomorphism if and only if $d= \pm 1$. Finally, we shall take $d \theta=d \theta_{1} \wedge \ldots \wedge d \theta_{n}$ as the standard volume form on $T^{n}$. A diffeomorphism $\phi$ of $T^{n}$ is said to be volume-preserving whenever $\phi$ preserves $d \theta$.

Return to a fibering (1.3) with $M=T^{n}$. Assume that the fibering has connected fibers. Then

$$
\mu(\theta)=d_{1} \theta_{1}+\ldots+d_{n} \theta_{n}+h(\theta),
$$

where $d_{1}, \ldots, d_{n}$ are relatively prime integers, and $h$ is a $2 \pi$-periodic function. By a volume-preserving bundle map, we may assume that

$$
\mu(\theta)=\theta_{1}+h(\theta) .
$$

We now restrict ourselves to the case $n=2$. Then the fibering $\tau: T^{2} \rightarrow$ $S^{1}$ is a circle bundle on $S^{1}$. From the bundle classification theorem (see [15], p. 97) and the isotropy classification of $\operatorname{Diff}\left(S^{1}\right)$ (see [8], p. 186), one knows that there are only two inequivalent classes of circle bundles over $S^{1}$. Since the total space $T^{2}$ is orientable, then $\tau: T^{2} \rightarrow S^{1}$ is a trivial circle bundle. This implies that there is a smooth bundle map

$$
\Phi=\left(\tau_{1}, \tau_{2}\right): T^{2} \rightarrow S^{1} \times S^{1}
$$

with $\tau=\tau_{1}$.

The following result is equivalent to the fact that an analytic circle bundle $T^{2} \rightarrow S^{1}$ is analytically trivial. For the lack of references, we shall give a proof by using the smooth trivialization $\Phi$.

Proposition 2.1. Let $\mu$ be given by (2.3) in which $h(\theta)$ is an analytic $2 \pi$-periodic function. Assume that d $\mu$ vanishes nowhere on $T^{n}$. If $n=2$, then there is an analytic diffeomorphism $\Psi$ of $T^{2}$ such that for a lifting $\widetilde{\Psi}, \mu \circ \widetilde{\Psi}(\theta)=\theta_{1}$. 
Proof. We shall modify $\tau_{2}$ in (2.4) to get an analytic bundle map $\Psi$. To this end, we put the lifting $\Phi$ of $\Phi$ in the form (2.2) with $n=2$. By truncating the Fourier series of $f_{2}$, we can find a sequence of analytic functions $g_{k}(\theta)$ such that for $k \rightarrow \infty$,

$$
\left\|g_{k}-f_{2}\right\|, \quad\left\|\partial_{\theta_{j}}\left(g_{k}-f_{2}\right)\right\| \rightarrow 0,
$$

where $\|\cdot\|$ stands for the $L^{\infty}$-norm on $\mathbb{R}^{n}$. We now consider the mapping $\Phi_{k}: T^{2} \rightarrow T^{2}$ defined by

$$
\left(e^{i \theta_{1}}, e^{i \theta_{2}}\right) \rightarrow\left(\tau_{1}(\theta), e^{i\left(d_{2,1} \theta_{1}+d_{2,2} \theta_{2}+g_{k}(\theta)\right)}\right) .
$$

We shall denote by $D \Phi$ the Jacobian matrix of $\Phi$. Obviously, $D \Phi_{k} \rightarrow$ $D \Phi$ uniformly on $T^{2}$ as $k \rightarrow \infty$. Hence, $\Phi_{k}$ is a local diffeomorphism for large $k$. Since $\Phi$ and $\Phi_{k}$ are of the same degree, then $\Phi_{k}$ is a diffeomorphism for large $k$. Furthermore, they also have the same first component. Thus, for $k$ large, $\Phi_{k}$ is an analytic trivialization for the fibering $\tau: T^{2} \rightarrow S^{1}$, which gives us

$$
\mu \circ \Phi_{k}^{-1}(\theta) \equiv \theta_{1}(\bmod 2 \pi) .
$$

Take $\Psi=\Phi_{k}^{-1}$. Then, for a suitable lifting $\widetilde{\Psi}$, one has $\mu \circ \widetilde{\Psi}(\theta)=\theta_{1}$. This completes the proof of Proposition 2.1.

The annulus $A_{r}$ has a universal covering

$$
S_{r} \subset \mathbb{C}^{n}:\left|\operatorname{Im} \theta_{j}\right|<r, \quad 1 \leq j \leq n,
$$

for which the covering mapping is given by (2.1). We have the following.

Theorem 2.2. Let $h$ be a $2 \pi$-periodic holomorphic function in $S_{r}$, and let $\mu$ be defined by (2.3). Assume that $h(\theta)$ is real for $\theta \in \mathbb{R}^{n}$. Then there is a constant $\epsilon>0$, which is independent of $r$, such that for $0<r<1$ and

$$
\|h\|_{r}=\sup _{\theta \in S_{r}}\{|h(\theta)|\} \leq \epsilon r^{3},
$$

there exist a unique $2 \pi$-periodic function $k\left(\theta_{1}\right)$ and a volume-preserving analytic transformation $\Phi$ of $T^{n}$ such that for a lifting $\widetilde{\Phi}$,

$$
\mu \circ \widetilde{\Phi}(\theta)=\theta_{1}+k\left(\theta_{1}\right)
$$

with $\int_{0}^{2 \pi} k\left(\theta_{1}\right) d \theta_{1}=0$.

Proof. The existence of $\Phi$ will be given in section 4 by a KAM argument. Here, we only verify the uniqueness of the invariant function $k$. Let $\psi$ be a volume-preserving analytic transformation of $T^{n}$, and $\theta^{*}=\widetilde{\psi}(\theta)$ a lifting satisfying

$$
\theta_{1}^{*}+\hat{k}\left(\theta_{1}^{*}\right)=\theta_{1}+k\left(\theta_{1}\right)
$$


for a $2 \pi$-periodic function $\hat{k}$ with $\int_{0}^{2 \pi} \hat{k}\left(\theta_{1}\right) d \theta_{1}=0$. We need to show that $\hat{k}=k$. One first notice that $1+k^{\prime}$ and $1+\hat{k}^{\prime}$ are positive on $R^{1}$, since $d \mu \neq 0$ on $T^{n}$. Hence, (2.8) implies that the first component of $\widetilde{\psi}$ can be written as

$$
\theta_{1}^{*}=\theta_{1}+f_{1}\left(\theta_{1}\right)
$$

for some $2 \pi$-periodic function $f_{1}$. The rest of components of $\widetilde{\psi}$ can be put into

$$
\theta_{\alpha}^{*}=\sum_{\beta=1}^{n} d_{\alpha, \beta} \theta_{\beta}+f_{\alpha}(\theta), \quad 2 \leq \alpha \leq n,
$$

in which $d_{\alpha, \beta}$ are integers, and $f_{\alpha}$ are $2 \pi$-periodic functions.

Denote

$$
\theta_{\alpha}^{\prime}=\sum_{\beta \geq 2} d_{\alpha, \beta} \theta_{\beta}, \quad \alpha \geq 2
$$

Also, define $d^{\prime}$ by

$$
d^{\prime} h(\theta)=\sum_{\alpha=2}^{n} \partial_{\theta_{\alpha}} h(\theta) d \theta_{\alpha}
$$

Since $\widetilde{\psi}$ is volume-preserving, then

$$
1 \equiv\left(1+f_{1}^{\prime}\left(\theta_{1}\right)\right) \operatorname{det} \frac{\partial\left(\theta_{2}^{\prime}+f_{2}(\theta), \ldots \theta_{n}^{\prime}+f_{n}(\theta)\right)}{\partial\left(\theta_{2}, \ldots, \theta_{n}\right)} .
$$

Notice that

$$
d^{\prime}\left(\theta_{2}^{\prime}+f_{2}(\theta)\right) \wedge \ldots \wedge d^{\prime}\left(\theta_{n}^{\prime}+f_{n}(\theta)\right)=d^{\prime} \theta_{2}^{\prime} \wedge \ldots \wedge d^{\prime} \theta_{n}^{\prime}+\ldots,
$$

where the term omitted is an exact $(n-1)$-form in the variables $\theta_{2}, \ldots, \theta_{n}$. Thus

$$
\int_{0 \leq \theta_{2}, \ldots, \theta_{n} \leq 2 \pi} d^{\prime}\left(\theta_{2}^{\prime}+f_{2}(\theta)\right) \wedge \ldots \wedge d^{\prime}\left(\theta_{n}^{\prime}+f_{n}(\theta)\right)
$$

is the total volume of $d^{\prime} \theta_{2}^{\prime} \wedge \ldots \wedge d^{\prime} \theta_{n}^{\prime}$, which is obviously independent of $\theta_{1}$. Averaging the right side of (2.9) over $0 \leq \theta_{\alpha} \leq 2 \pi$ for $2 \leq \alpha \leq n$, we see that $1+f_{1}^{\prime}\left(\theta_{1}\right)$ is constant. Hence, $f_{1} \equiv c$. Returning to (2.8), we get

$$
k\left(\theta_{1}\right)=c+\hat{k}\left(\theta_{1}+c\right) .
$$

By the assumption, we know that the average values of $k$ and $\hat{k}$ for $0 \leq \theta_{1} \leq 2 \pi$ vanish. Averaging the above over $0 \leq \theta_{1} \leq 2 \pi$, we finally get $c=0$. Therefore, $\hat{k}=k$. This proves the uniqueness of the function $k$. 
2.2. The case $n=1$. We consider an immersion $f: S^{1} \rightarrow \mathbb{C}$. Put

$$
\omega_{f}=\rho\left(\theta_{1}\right) e^{i\left(d \theta_{1}+h\left(\theta_{1}\right)\right)} d \theta_{1}, \quad d \in \mathbb{Z},
$$

where $h$ and $\rho$ are $2 \pi$-periodic functions with $\rho>0$. Obviously, $d=d_{f}$ is the Hopf degree of the Gauss map of $f$. One can see that the immersion $f: S^{1} \rightarrow \mathbb{C}$ is non-critical if and only if, locally, the immersion $f$ is strictly convex in $\mathbb{C}=\mathbb{R}^{2}$.

We have the following result.

Theorem 2.3 (Whitney-Graustein, [21]). Let $f_{0}$ and $f_{1}$ be two immersions of $S^{1}$ in $\mathbb{C}$. Then $f_{1}$ is regularly homotopic to $f_{0}$ if and only if $d_{f_{0}}=d_{f_{1}}$. Furthermore, if both immersions $f_{0}$ and $f_{1}$ are non-critical, then $f_{0}$ and $f_{1}$ are also regularly homotopic through a family of noncritical immersions.

Proof. The first part of the theorem is proved in [21. We now modify Whitney's proof to show the second part of the theorem. Put

$$
\omega_{f_{j}}=\rho_{j}\left(\theta_{1}\right) e^{i\left(d \theta_{1}+h_{j}\left(\theta_{1}\right)\right)}, \quad d_{f_{0}}=d=d_{f_{1}} .
$$

We have $d \neq 0$ and

$$
1+\frac{1}{d} h_{j}^{\prime}\left(\theta_{1}\right)>0
$$

We now consider an orientation-preserving transformation $\psi_{j}$ of $S^{1}$ given by

$$
\psi_{j}: e^{i \theta_{1}} \rightarrow e^{i\left(\theta_{1}+h_{j}\left(\theta_{1}\right) / d\right)}, \quad j=0,1 .
$$

Obviously, $f_{j} \circ \psi_{j}^{-1}$ is regularly homotopic to $f_{j}$. Hence, we may still denote $f_{j} \circ \psi_{j}^{-1}$ by $f_{j}$. Setting $\widetilde{f}_{j}\left(\theta_{1}\right)=f_{j}\left(e^{i \theta_{1}}\right)$, we then have

$$
\tilde{f}_{j}^{\prime}\left(\theta_{1}\right)=\rho_{j}\left(\theta_{1}\right) e^{i d \theta_{1}}, \quad \rho_{j}\left(\theta_{1}\right)>0, \quad j=0,1 .
$$

Put

$$
g_{t}\left(\theta_{1}\right)=\left((1-t) \rho_{0}\left(\theta_{1}\right)+t \rho_{1}\left(\theta_{1}\right)\right) e^{i d \theta_{1}}, \quad 0 \leq t \leq 1
$$

Obviously, the average value of $g_{t}\left(\theta_{1}\right)$ on $S^{1}$ vanishes for all $t$. We may assume that the average value of $\widetilde{f}_{j}$ vanishes for $j=0,1$. Let $\tilde{f}_{t}$ be the unique $2 \pi$-periodic function in $\theta_{1}$ such that its average value for $0 \leq \theta_{1} \leq 2 \pi$ is zero, and $\widetilde{f}_{t}^{\prime}=g_{t}$. It is clear that $\widetilde{f}_{t}$ is a non-critical immersion for all $t$. Now $f_{t}\left(e^{i \theta_{1}}\right)=\widetilde{f}_{t}\left(\theta_{1}\right)$ gives us a regular homotopy $f_{t}$ of non-critical immersions from $f_{0}$ to $f_{1}$. The proof of Theorem 2.3 is complete.

For later use, we remark that a non-critical immersion $f: S^{1} \rightarrow \mathbb{C}$ is an embedding if and only if $d_{f}= \pm 1$. Here, we need a result of Whitney [21], which says that

$$
I_{f} \equiv d_{f}-\operatorname{sign} d_{f}
$$


is the algebraic sum of double points when $f$ is an immersion with only double points in normal crossings. It is clear that the Whitney selfintersection number $I_{f}$ must vanish if $f$ is an embedding. Conversely, if $f$ is non-critical and $d= \pm 1$, one can see that the Gauss map

$$
z \rightarrow-i \frac{f^{\prime}(z)}{\left|f^{\prime}(z)\right|}
$$

is a diffeomorphism of $S^{1}$. Therefore, $f$ is an embedding.

\section{ThE NORMAL FORM OF TOTALLY REAL TORI}

In this section, we shall first prove a result of Moser about normalizing analytic volume elements on a compact manifold $M$. We shall also give some relevant estimates when $M=T^{n}$. Finally, we shall give a proof for Theorem 1.3 by using Theorem 2.2.

3.1. A theorem of Moser. Consider a non-critical totally real immersion $\varphi: M \rightarrow \mathbb{C}^{n}$. The pull-back $\omega_{\varphi}$ is a complex-valued $n$-form on $M$. In local coordinates $x=\left(x_{1}, \ldots, x_{n}\right)$, we put

$$
\omega_{\varphi}(x)=\rho(x) e^{i \mu(x)} d x_{1} \wedge \ldots \wedge d x_{n},
$$

where $\rho(x)$ is a positive function and $\mu(x)(\bmod 2 \pi)$ is a real function. Define

$$
\nu(x)=\rho(x) d x_{1} \wedge \ldots \wedge d x_{n} .
$$

When $M$ is orientable, we require that $\left(x_{1}, \ldots, x_{n}\right)$ are the coordinates which agree with a fixed orientation. Then $\nu$ is a volume form on $M$. When $M$ is non-orientable, $\nu$ is still globally defined. However, $\nu$ is not an $n$-form on $M$; instead, it is a positive $n$-form of odd kind. In both cases, $\nu$ is called a volume element on $M$.

We have the following result.

Theorem 3.1 (Moser [11). Let $\nu_{0}$ and $\nu_{1}$ be two analytic volume elements on a compact manifold $M$ with $\int_{M} \nu_{0}=\int_{M} \nu_{1}$. Then there is an analytic diffeomorphism $\phi$ of $M$ such that $\phi^{*} \nu_{1}=\nu_{0}$.

Proof. The proof given in [11] is only for smooth volume elements. However, it works equally well in the analytic case. Let us choose an analytic Riemannian metric on $M$. Then the Hodge decomposition theorem gives us

$$
\nu_{j}=d \delta \beta_{j}+h_{j}, \quad j=1,2,
$$

where $h_{j}$ is a harmonic $n$-form. Since $\beta_{j}$ is of top degree, we can rewrite

$$
\nu_{j}=\Delta \beta_{j}+h_{j} .
$$


Now the regularity of the Laplace-Beltrami operator $\Delta$ implies that $h_{j}$ and $\beta_{j}$ are analytic $n$-forms (see [12], p. 177). Since $\int_{M} \nu_{1}=\int_{M} \nu_{0}$, then $h_{1}=h_{0}$. Let

$$
\nu_{t}=(1-t) \nu_{0}+t \nu_{1}
$$

Then we have

$$
\nu_{t}=d \alpha_{t}+h_{0}
$$

where $\alpha_{t}=(1-t) \delta \beta_{0}+t \delta \beta_{1}$. Obviously, $\alpha_{t}$ is a family of analytic $n$-forms depending analytically on the parameter $t$. Using $\alpha_{t}$, one can construct a family of analytic diffeomorphisms $\phi_{t}$ such that $\phi_{t}^{*} \nu_{t}=\nu_{0}$. For the detail, we refer to [11.

The above proof does not provide us any estimate for the mapping $\phi$. For the proof of Theorem 1.3, we shall give some estimates of $\phi$ for the case $M=T^{n}$.

Let $h(\theta)$ be a $2 \pi$-periodic holomorphic function defined in $S_{r}$. We shall introduce a useful decomposition

$$
h(\theta)=\sum_{j=0}^{n}\left(L_{j} h\right)(\theta),
$$

in which $L_{0} h$ is a constant, and $L_{j} h$ depends only on $\theta_{1}, \ldots, \theta_{j}$. To ensure the uniqueness of the decomposition, we require that

$$
\left[L_{j} h\right]_{j}=0, \quad 1 \leq j \leq n,
$$

in which and also in the later discussion, we use the following notations

$$
[f]_{j}=\frac{1}{2 \pi} \int_{0}^{2 \pi} f(\theta) d \theta_{j}, \quad[f]=\frac{1}{(2 \pi)^{n}} \int_{0}^{2 \pi} \cdots \int_{0}^{2 \pi} f(\theta) d \theta .
$$

Now, the condition (3.1) implies that

$$
L_{0} h(\theta)+\ldots+L_{j} h(\theta)=\left[\ldots[h]_{j+1} \ldots\right]_{n}, \quad 0 \leq j<n .
$$

In particular, $L_{0} h$ is the constant term of the Fourier series of $h$. Obviously, we have

$$
\left\|L_{0} h+\ldots+L_{j} h\right\|_{r} \leq\|h\|_{r}
$$

Therefore, we get

$$
\left\|L_{j} h\right\|_{r} \leq 2\|h\|_{r}, \quad 0 \leq j \leq n .
$$

Denote

$$
D_{j} h(\theta)=\partial_{\theta_{j}} h(\theta) .
$$

We also define $D_{j}^{-1} h(\theta)$ to be the unique anti-derivative of $h$ with respect to the variable $\theta_{j}$ which satisfies the normalizing condition

$$
\left[D_{j}^{-1} h\right]_{j}=0 .
$$


In addition, if $h(\theta)$ satisfies

$$
[h]_{j}=0,
$$

then $D_{j}^{-1} h$ is also $2 \pi$-periodic, and

$$
D_{j} D_{j}^{-1} h=D_{j}^{-1} D_{j} h=h .
$$

We need the following.

Lemma 3.2. Let $h$ be a $2 \pi$-periodic holomorphic function in $S_{r}$ satisfying (3.4). Then

$$
\left\|D_{j}^{-1} h\right\|_{r} \leq 2 \pi\|h\|_{r} .
$$

Proof. We fix $\xi \in S_{r}$ and put $\xi_{j}=t+i s$. We also let $\theta_{k}=\xi_{k}$ for $k \neq j$. Since $D_{j}^{-1} h$ is $2 \pi$-periodic in $\theta_{j}$, then (3.3) implies that

$$
\int_{\xi_{j}-\pi}^{\xi_{j}+\pi} D_{j}^{-1} h(\theta) d \theta_{j}=0 .
$$

Hence, there is $t_{0} \in(t-\pi, t+\pi)$ such that $\operatorname{Re} D_{j}^{-1} h(\theta)=0$ for $\theta_{j}=$ $t_{0}+i s$. We now have

$$
\operatorname{Re} D_{j}^{-1} h(\xi)=\int_{t_{0}+i s}^{t+i s} \operatorname{Re} h(\theta) d \theta_{j} .
$$

Thus, we get

$$
\left|\operatorname{Re} D_{j}^{-1} h(\xi)\right| \leq \pi\|h\|_{r} .
$$

One can also obtain that $\left|\operatorname{Im} D_{j}^{-1} h(\xi)\right| \leq \pi\|h\|_{r}$. Therefore, (3.5) is verified.

Consider a transformation $\phi$ given by

$$
\theta_{j}^{\prime}=\theta_{j}+f_{j}(\theta), \quad j=1, \ldots n,
$$

where each $f_{j}$ is $2 \pi$-periodic on $S_{r}$. We first prove the following result.

Lemma 3.3. Let $\phi: S_{r} \rightarrow \mathbb{C}^{n}$ be a mapping with the form (3.6). Assume that

$$
\|f\|_{r} \leq \frac{r}{4 n}
$$

Then for $0<r<1$, one has

$$
\phi: S_{r / 2} \rightarrow S_{r}, \quad \phi^{-1}: S_{r / 4} \rightarrow S_{r / 2} .
$$

Proof. Obviously, 3.7) implies that $\phi: S_{r / 2} \rightarrow S_{r}$. For the inverse mapping, we fix $\theta \in S_{r / 4}$ and consider a mapping

$$
T: \theta^{\prime} \rightarrow \theta-f\left(\theta^{\prime}\right)
$$


From (3.7), it is clear that $T: S_{r / 2} \rightarrow S_{r / 2}$. By Cauchy inequalities, we have

Hence

$$
\left\|D_{k} f_{j}\right\|_{r / 2} \leq \frac{1}{2 n}
$$

$$
\|D T\|_{r / 2} \leq 1 / 2
$$

where and also in the sequel, the operator norm $\|D f\|_{r}$ of a holomorphic mapping

is defined by

$$
f=\left(f_{1}, \ldots, f_{m}\right): S_{r} \rightarrow \mathbb{C}^{m}
$$

$$
\|D f\|_{r}=\sup _{1 \leq j \leq m}\left\{\sum_{k}\left|D_{k} f_{j}(\theta)\right| ; \theta \in S_{r}\right\} .
$$

Therefore, we know that $T: S_{r / 2} \rightarrow S_{r / 2}$ is a contraction mapping. Now the unique fixed point $\theta^{\prime} \in S_{r / 2}$ of $T$ is precisely the inverse image $\phi^{-1}(\theta)$. The proof of Lemma 3.3 is complete.

We have the following estimates.

Proposition 3.4. Let $\omega=(1+b(\theta)) d \theta$ be a real $n$-form on $R^{n}$. Assume that $b(\theta)$ is a $2 \pi$-periodic holomorphic function on $S_{r}$ with

$$
\|b\|_{r} \leq \frac{r}{32 n \pi}, \quad 0<r<1 .
$$

Then there is a holomorphic mapping $\phi$ in the form (3.6) such that $\phi\left(\mathbb{R}^{n}\right)=\mathbb{R}^{n}$ and $\omega=(1+[b]) \phi^{*} d \theta$. Furthermore, $\phi$ satisfies (3.8), and

$$
\|f\|_{r} \leq 8 \pi\|b\|_{r} .
$$

Proof. We shall seek a transformation $\phi$ in the form (3.6) such that $f_{j}(\theta)$ depends only on $\theta_{1}, \ldots, \theta_{j}$. Then $\omega=(1+[b]) \phi^{*} d \theta$ can be rewritten as

$$
(1+[b])\left(1+D_{1} f_{1}(\theta)\right) \ldots\left(1+D_{n} f_{n}(\theta)\right)=1+b(\theta) .
$$

We further require that

$$
\left[f_{j}\right]_{j}=0 .
$$

Thus, by applying $[\cdot]_{k}(k>j)$ to (3.11), we get

$$
(1+[b])\left(1+D_{1} f_{1}(\theta)\right) \ldots\left(1+D_{j} f_{j}(\theta)\right)=1+\sum_{l=0}^{j} L_{l} b(\theta)
$$

for $1 \leq j \leq n$. Hence,

$$
D_{j} f_{j}(\theta)=\frac{L_{j} b(\theta)}{1+\sum_{l=0}^{j-1} L_{l} b(\theta)}
$$


From the normalizing condition (3.12), it follows that

$$
f_{j}(\theta)=\frac{D_{j}^{-1} L_{j} b(\theta)}{1+\sum_{l=0}^{j-1} L_{l} b(\theta)} .
$$

Using (3.2), (3.5) and (3.9), we obtain

$$
\left\|f_{j}\right\|_{r} \leq \frac{4 \pi\|b\|_{r}}{1-2 n\|b\|_{r}} \leq 8 \pi\|b\|_{r} .
$$

From (3.9) again, we see that (3.7) is satisfied. Thus, Lemma 3.3 implies that (3.8) holds. This completes the proof of Proposition 3.4.

3.2. Proof of Theorem 1.3. Let $M$ and $\varphi$ be as in Theorem 1.3. We take

$$
r=r_{0} / 2, \quad 0<r_{0}<1
$$

Assume that

$$
\epsilon_{0}<\frac{1}{8 e^{2} n^{2}}
$$

From Cauchy inequalities, we get

$$
\|D \varphi-\mathrm{Id}\|_{r} \leq n \frac{\|\varphi-\mathrm{Id}\|_{r_{0}}}{e^{-r_{0} / 2}-e^{-r_{0}}} \leq \frac{2 n e}{r_{0}}\|\varphi-\mathrm{Id}\|_{r_{0}} \leq 2 e n \epsilon_{0} r_{0}^{3}
$$

in which the last inequality is obtained from (1.4). Let $\lambda_{1}(z), \ldots, \lambda_{n}(z)$ be the eigenvalues of $D \varphi(z)$. Then the above estimate gives us

$$
\left|\lambda_{j}(z)-1\right| \leq 2 e n \epsilon_{0} r_{0}^{3} \text {. }
$$

Noticing that

$$
|\log (1+x)| \leq 2|x|
$$

for $|x| \leq 1 / 2$, we get from 3.13 ) that

$$
\left|\sum_{j=1}^{n} \log \lambda_{j}(z)\right| \leq 4 e n^{2} \epsilon_{0} r_{0}^{3}, \quad z \in A_{r} .
$$

Now, using the inequality

$$
\left|e^{w}-1\right| \leq e|w|
$$

for $|w| \leq 1$, we obtain

$$
\left|\lambda_{1}(z) \ldots \lambda_{n}(z)-1\right|=\left|e^{\log \lambda_{1}(z) \ldots \lambda_{n}(z)}-1\right| \leq 4 e^{2} n^{2} \epsilon_{0} r_{0}^{3}
$$

for $z \in A_{r}$. Put

$$
1+a(z) \equiv \operatorname{det}(D \varphi(z))=\lambda_{1}(z) \ldots \lambda_{n}(z) .
$$


Then

$$
\|a\|_{r} \leq 4 e^{2} n^{2} \epsilon_{0} r^{3}
$$

Let $\widetilde{\omega_{\varphi}}=\pi^{*} \omega_{\varphi}$. Put

$$
\begin{aligned}
& b_{1}(\theta)=\{(1+a(z))(1+\overline{a(\bar{z})})\}^{1 / 2}-1, \\
& h_{1}(\theta)=\frac{1}{2 i}\{\log (1+a(z))-\log (1+\overline{a(\bar{z})})\}
\end{aligned}
$$

with $z=\left(e^{i \theta_{1}}, \ldots, e^{i \theta_{n}}\right)$ and $\bar{z} \equiv\left(e^{i \bar{\theta}_{1}}, \ldots, e^{i \bar{\theta}_{n}}\right)$. Then, $b_{1}$ and $h_{1}$ are holomorphic functions in $S_{r}$. On $\mathbb{R}^{n}$, we now have a decomposition

$$
\widetilde{\omega_{\varphi}}=i^{n} \nu_{1} e^{i \mu_{1}}
$$

with

$$
\nu_{1}(\theta)=\left(1+b_{1}(\theta)\right) d \theta, \quad \mu_{1}(\theta)=\theta_{1}+\ldots+\theta_{n}+h_{1}(\theta) .
$$

From (3.13), (3.14) and (3.16), we obtain

$$
|\log (1+a(z))| \leq 8 e^{2} n^{2} \epsilon_{0} r_{0}^{3}, \quad|\log (1+\overline{a(\bar{z})})| \leq 8 e^{2} n^{2} \epsilon_{0} r_{0}^{3}
$$

for $z \in A_{r}$. Hence, from (3.13) and (3.15), it follows that

$$
\left\|b_{1}\right\|_{r} \leq c_{0} \epsilon_{0} r^{3}, \quad\left\|h_{1}\right\|_{r} \leq c_{0} \epsilon_{0} r^{3}
$$

in which and also in the sequel, we denote by $c_{j}(j=0,1, \ldots)$ a constant which depends only on $n$, but is larger than 1 .

Consider the linear transformation

$$
\phi_{0}: \theta_{1}^{\prime}=\theta_{1}+\ldots+\theta_{n}, \quad \theta_{j}^{\prime}=\theta_{j}, \quad j \geq 2 .
$$

Put $\mu_{2}=\mu_{1} \circ \phi_{0}^{-1}$ in the form

$$
\mu_{2}(\theta)=\theta_{1}+h_{2}(\theta), \quad h_{2}(\theta)=h_{1} \circ \phi_{0}^{-1}(\theta) .
$$

Let

$$
\nu_{2}=\nu_{1} \circ \phi_{0}^{-1}=\left(1+b_{2}\right) d \theta, \quad b_{2}=b_{1} \circ \phi_{0}^{-1} .
$$

Notice that the domain $S_{r}$ is invariant by $\phi_{0}$. Then (3.17) gives us

$$
\left\|b_{2}\right\|_{r} \leq c_{0} \epsilon_{0} r^{3}, \quad\left\|h_{2}\right\|_{r} \leq c_{0} \epsilon_{0} r^{3} .
$$

We now choose $\epsilon_{0}$ so small that (3.18) implies that (3.9) holds for $b=b_{2}$. Let $\phi_{1}$ be the transformation given in Proposition 3.4. Then

$$
\left(\phi_{1}^{-1}\right)^{*} \nu_{2}=i^{n}\left(1+\left[b_{3}\right]\right) d \theta .
$$

Notice that $\phi_{0}$ and $\phi_{1}$ are volume-preserving. Hence, $\left[b_{3}\right]=[a]$. By setting

we then have

$$
\mu_{2} \circ \phi_{1}^{-1}(\theta)=\theta_{1}+h_{3}(\theta),
$$

$$
h_{3}\left(\phi_{1}(\theta)\right)=h_{2}(\theta)-f_{1}(\theta) .
$$


Notice that $\phi_{1}^{-1}: S_{r / 4} \rightarrow S_{r / 2}$. Thus

$$
\left\|h_{3}\right\|_{r / 4} \leq\left\|h_{2}\right\|_{r / 2}+\left\|f_{1}\right\|_{r / 2}
$$

Together with (3.10) and (3.17), we get

$$
\left\|h_{3}\right\|_{r / 4} \leq c_{1} \epsilon_{0} r^{3} \text {. }
$$

Now for a possibly smaller $\epsilon_{0}$, (3.19) implies that $h_{3}$ satisfies (2.6). Thus, by applying Theorem 2.2, we obtain a volume-preserving transformation $\phi_{2}$ such that

$$
\phi_{2}^{*} e^{i\left(\theta_{1}+h_{3}(\theta)\right)} d \theta=e^{i\left(\theta_{1}+k\left(\theta_{1}\right)\right)} d \theta .
$$

Put $\phi=\phi_{0}^{-1} \phi_{1}^{-1} \phi_{2} \phi_{0}$. Then, we obtain

$$
\phi^{*} \widetilde{\omega_{\varphi}}=i^{n}(1+[a]) e^{i\left(\theta_{1}+\ldots \theta_{n}+k\left(\theta_{1}+\ldots \theta_{n}\right)\right)} d \theta .
$$

To construct $g$ as stated in Theorem 1.3, we first notice that $\phi^{*} \widetilde{\omega_{\varphi}}$ is exact, i.e.

$$
\int_{T^{n}} e^{i\left(\theta_{1}+\ldots \theta_{n}+k\left(\theta_{1}+\ldots \theta_{n}\right)\right)} d \theta=0 .
$$

Equivalently, this means that the average value of $e^{i\left(\theta_{1}+k\left(\theta_{1}\right)\right)}$ for $0 \leq$ $\theta_{1} \leq 2 \pi$ vanishes. Hence, there is an immersion

$$
g: S^{1} \rightarrow \mathbb{C}
$$

such that

$$
\frac{d}{d \theta_{1}} g\left(e^{i \theta_{1}}\right)=e^{i\left(\theta_{1}+k\left(\theta_{1}\right)\right)}
$$

We further notice that $\varphi \circ \phi$ is a non-critical totally real immersion. Hence, from the right side of $(3.20)$, we see that $1+k^{\prime}\left(\theta_{1}\right) \neq 0$. Therefore, $g$ is a non-critical immersion with $d_{g}=1$. As we have seen in the end of section 2, this implies that $g$ is an embedding. Now, one can verify that the mapping $\psi$ given in Theorem 1.3 defines an embedding of $T^{n}$ with $\psi^{*} \Omega=\phi^{*} \varphi^{*} \Omega$. Let $\widetilde{T}^{n}=\psi\left(T^{n}\right)$. Then the analytic diffeomorphism $\Phi=\psi \circ \phi^{-1} \circ \varphi^{-1}: M \rightarrow \widetilde{T}^{n}$ satisfies

$$
\left.\Phi^{*} \Omega\right|_{\widetilde{T}^{n}}=\left.\Omega\right|_{M}
$$

By complexifying $\Phi$, we get a unimodular transformation defined near $M$ which transforms $M$ into $\widetilde{T}^{n}$.

Finally, we need to verify that up to a translation $\theta_{1} \rightarrow \theta_{1}+\pi$, the function $k\left(\theta_{1}\right)$ is uniquely determined by $M$. More precisely, we want to show that if $M$ is unimodularly equivalent to an analytic embedding of $T^{n}$ given by another mapping

$$
\hat{\psi}:\left(z_{1}, z^{\prime}\right) \rightarrow\left(\zeta^{-1} \hat{g}\left(\zeta z_{1}\right), z^{\prime}\right)
$$


with

$$
\frac{d}{d \theta_{1}} \hat{g}\left(e^{i \theta_{1}}\right)=\hat{\rho}_{0} e^{i \hat{k}\left(\theta_{1}\right)}, \quad[\hat{k}]=0
$$

Then, either $\hat{k}=k$, or $\hat{k}\left(\theta_{1}+\pi\right)=k\left(\theta_{1}\right)$. For the proof, we notice that

$$
\omega_{\hat{\psi}}=\hat{\rho}_{0} e^{i\left(\theta_{1}+\ldots+\theta_{n}+\hat{k}\left(\theta_{1}+\ldots+\theta_{n}\right)\right)} .
$$

If $M$ is unimodularly equivalent to $\hat{\psi}\left(T^{n}\right)$, then there is a diffeomorphism $\phi$ of $T^{n}$ such that $\phi^{*} \omega_{\hat{\psi}}=\omega_{\psi}$. In brief, let us still denote the lifting of $\phi$ by $\phi$. This implies that either

$$
\hat{\rho}_{0} \phi^{*} d \theta=\rho_{0} d \theta
$$

and

$$
\hat{K} \circ \phi(\theta)=K(\theta)+2 d \pi, \quad d \in \mathbb{Z}
$$

for $K(\theta)=\theta_{1}+k\left(\theta_{1}\right)$ and $\hat{K}(\theta)=\theta_{1}+\hat{k}\left(\theta_{1}\right)$; or

$$
\hat{\rho}_{0} \phi^{*} d \theta=-\rho_{0} d \theta
$$

and

$$
\hat{K} \circ \phi(\theta)=K(\theta)+(2 d+1) \pi .
$$

If (3.21) holds, then we see that $\hat{\rho}_{0}=\rho_{0}$ and $\phi$ is volume-preserving. Let $L_{a}$ be the translation

$$
\theta_{1} \rightarrow \theta_{1}+a, \quad \theta_{j} \rightarrow \theta_{j}, \quad j \geq 2 .
$$

Notice that $k$ is $2 \pi$-periodic. Then $(3.22)$ implies that $\hat{K} \circ \phi \circ L_{-2 d \pi}=K$. Since $\phi \circ L_{-2 d \pi}$ is volume-preserving, then Theorem 2.2 implies that $\hat{k}=k$. Next, we assume that (3.23) holds. In this case, we get $\hat{\rho}_{0}=\rho_{0}$ and $\phi^{*} d \theta=-d \theta$. Here we use the assumption $n \geq 2$. We consider the volume-preserving mapping

$$
\hat{\phi}(\theta)=\phi\left(\theta_{1}, \ldots, \theta_{n-1},-\theta_{n}\right) .
$$

Notice that $\hat{K}$ depends only $\theta_{1}$. Hence, we still have

$$
\hat{K} \circ \hat{\phi}(\theta)=K(\theta)+(2 d+1) \pi .
$$

Now (3.24) can be rewritten as $\hat{K} \circ \hat{\phi} \circ L_{(2 d+1) \pi}=\theta_{1}+k\left(\theta_{1}+\pi\right)$. Again, Theorem 2.2 implies that $\hat{k}\left(\theta_{1}\right)=k\left(\theta_{1}+\pi\right)$. This completes the proof of Theorem 1.3 . 


\section{Normalizing FUnCTIONS With VOLUME-PRESERVING MAPPINGS}

This section is devoted to the proof of Theorem 2.2. We shall construct volume-preserving transformations of $T^{n}$ through the time-1 mappings of divergent free vector fields, since the latter are easier to be handled because of the linearity of the compatibility condition. We shall accomplish the proof of Theorem 2.2 by using a KAM argument.

4.1. Some estimates for flows. Consider the flow $\phi_{t}$ determined by a system of ordinary differential equations

$$
\frac{d \theta_{j}}{d t}=p_{j}(\theta), \quad 1 \leq j \leq n
$$

Set

$$
\phi_{t}: \theta_{j}^{\prime}=\theta_{j}+f_{j}(\theta, t), \quad 1 \leq j \leq n .
$$

For $p=\left(p_{1}, \ldots, p_{n}\right)$, we put

$$
\|p\|_{r}=\sup \left\{\left|p_{j}(\theta)\right| ; \theta \in S_{r}, 1 \leq j \leq n\right\} .
$$

We need the following result.

Lemma 4.1. Let $p_{1}, \ldots, p_{n}$ be holomorphic functions on $S_{r_{1}}$. Assume that

$$
\|p\|_{r_{1}} \leq r_{1} \delta
$$

Then for $-1 \leq t \leq 1$ and $0<\delta<1 / 2$, one has

$$
\|f(\cdot, t)\|_{(1-\delta) r_{1}} \leq\|p\|_{r_{1}}
$$

and

$$
\left\|f_{j}(\cdot, t)-p_{j}(\cdot) t\right\|_{(1-2 \delta) r_{1}} \leq \frac{n\|p\|_{r_{1}}^{2}}{r_{1} \delta} .
$$

Furthermore, if all $p_{j}$ are $2 \pi$-periodic, then $f_{j}(\theta, t)$ are also $2 \pi$-periodic in $\theta$ for fixing $|t| \leq 1$.

Proof. We first want to prove that $\phi_{t}(\theta)$ is holomorphic for $\theta \in S_{(1-\delta) r_{1}}$ and $|t| \leq 1$. By the Cauchy existence theorem, it suffices to show that for any $t$ with $|t| \leq 1$, one has

$$
\phi_{t}\left(S_{(1-\delta) r_{1}}\right) \subset S_{r_{1}} .
$$

Otherwise, there are $0<\left|t_{0}\right| \leq 1$ and $\theta_{0} \in S_{(1-\delta) r_{1}}$ such that for some $k$

$$
\left|f_{k}\left(\theta_{0}, t\right)\right|<\left|f_{k}\left(\theta_{0}, t_{0}\right)\right|=\delta r_{1}, \quad|t|<\left|t_{0}\right|
$$


Rewrite (4.1) as

$$
f_{j}(\theta, t)=\int_{0}^{t} p_{j}\left(\phi_{\tau}(\theta)\right) d \tau, \quad j=1, \ldots, n .
$$

Then (4.2) implies that

$$
\left|f_{k}\left(\theta_{0}, t_{0}\right)\right|<\left\|p_{k}\right\|_{r_{1}} \leq r_{1} \delta
$$

which leads to a contradiction. Hence, we have verified that (4.5) holds. Using (4.6), we now obtain the inequality (4.3) for $|t| \leq 1$.

By Cauchy inequalities, we have

$$
\left\|D_{l} p_{k}\right\|_{(1-\delta) r_{1}} \leq \frac{\left\|p_{k}\right\|_{r_{1}}}{r_{1} \delta}
$$

Fix $\theta \in S_{(1-2 \delta) r_{1}}$ and $|t| \leq 1$. Then (4.3) and (4.5) give us

$$
\left|p_{k}(\theta+f(\theta, t))-p_{k}(\theta)\right| \leq \sum_{l=1}^{n}\left\|f_{l}(\cdot, t)\right\|_{(1-2 \delta) r_{1}} \cdot\left\|D_{l} p_{k}(\cdot, t)\right\|_{(1-\delta) r_{1}} .
$$

Together with (4.3) and (4.7), we get

$$
\left|p_{k}(\theta+f(\theta, t))-p_{k}(\theta)\right| \leq \frac{n\|p\|_{r_{1}}^{2}}{r_{1} \delta} .
$$

Now, from the identity

$$
f_{k}(\theta, t)-p_{k}(\theta) t=\int_{0}^{t}\left\{p_{k}(\theta+f(\theta, \tau))-p_{k}(\theta)\right\} d \tau
$$

we obtain the estimate (4.4). The periodicity of $f_{j}$ follows directly from the uniqueness of the solutions $f_{1}, \ldots, f_{n}$ to (4.1) and the assumption that all $p_{k}$ are $2 \pi$-periodic. The proof of Lemma 4.1 is complete.

We notice that if the vector field $v$ defined by (4.1) is divergent free, i.e.

$$
\sum_{j=1}^{n} D_{j} p_{j}(\theta)=0
$$

then $\phi_{t}$ is volume-preserving for all $t$.

\subsection{Approximate equations. We put}

$$
p_{1}=\frac{1}{1+D_{1} L_{1} h} \sum_{j=2}^{n} L_{j} h .
$$

In order that (4.8) is satisfied, we take

$$
p_{j}=D_{j}^{-1} D_{1}\left(\frac{-L_{j} h}{1+D_{1} L_{1} h}\right), \quad j=2, \ldots, n .
$$


Let $\phi_{t}$ the flow defined by (4.1). Set

$$
\phi=\phi_{-1}: \theta_{j}^{\prime}=\theta_{j}+f_{j}(\theta,-1) .
$$

With the above transformation, we now put

$$
\theta_{1}+k(\theta)=\theta_{1}^{\prime}+h\left(\theta^{\prime}\right) .
$$

Introduce the notations

$$
B_{r}=\max \left\{\left|L_{0} h\right|,\left\|D_{1} L_{1} h\right\|_{r}\right\}, \quad b_{r}=\max \left\{\left\|L_{2} h\right\|_{r}, \ldots,\left\|L_{n} h\right\|_{r}\right\} .
$$

We need the following.

Lemma 4.2. Let $p_{j}$ be defined by (4.9) and (4.10). Assume that

$$
B_{r} \leq 1 / 2 \text {. }
$$

Then there exists a constant $c_{2}$ such that for $0<r<1$ and $0<\delta<1 / 2$, one has

$$
\|p\|_{(1-\delta) r} \leq \frac{c_{2} b_{r}}{r \delta}
$$

Proof. Using Cauchy inequalities, we get

$$
\begin{gathered}
\left\|D_{1}^{2} L_{1} h\right\|_{(1-\delta) r} \leq \frac{B_{r}}{r \delta}, \\
\left\|D_{1} L_{j} h\right\|_{(1-\delta) r} \leq \frac{b_{r}}{r \delta}, \quad 2 \leq j \leq n .
\end{gathered}
$$

From (4.13) and (4.9), it follows that

$$
\left\|p_{1}\right\|_{(1-\delta) r} \leq 2 n b_{r} .
$$

We also have

$D_{1}\left(\frac{L_{j} h}{1+D_{1} L_{1} h}\right)=\frac{1}{\left(1+D_{1} L_{1}\right)^{2}}\left\{\left(1+D_{1} L_{1} h\right) D_{1} L_{j} h-D_{1}^{2} L_{1} h \cdot L_{j} h\right\}$.

Now, (3.5), (4.15) and (4.16) give us

$$
\left\|D_{j}^{-1} D_{1}\left(\frac{L_{j} h}{1+D_{1} L_{1} h}\right)\right\|_{(1-\delta) r} \leq 8 \pi\left(\left(1+B_{r}\right) \frac{b_{r}}{r \delta}+\frac{B_{r} b_{r}}{r \delta}\right) \leq \frac{16 \pi b_{r}}{r \delta},
$$

where the last inequality comes from (4.13). Together with (4.17), we can obtain (4.14) by taking $c_{2}=\max \{2 n, 16 \pi\}$.

To apply Lemma 4.1, we should take

$$
r_{1}=(1-\delta) r, \quad 0<r<1, \quad 0<\delta<1 / 4 .
$$

We also assume that

$$
b_{r} \leq \frac{r^{2} \delta^{2}}{n c_{2}}
$$


Notice that $r_{1}>r / 2$. Then, (4.14) and (4.18) imply that (4.2) holds. Since $(1-2 \delta) r<(1-\delta) r_{1}$, then (4.2) and (4.3) give us

$$
\|f(\cdot,-1)\|_{(1-2 \delta) r} \leq r \delta \text {. }
$$

From (4.4) and (4.14), we also have

$$
\left\|f_{j}(\cdot,-1)+p_{j}(\cdot)\right\|_{(1-3 \delta) r} \leq \frac{n c_{2}^{2} b_{r}^{2}}{r_{1} r^{2} \delta^{3}} \leq \frac{c_{3} b_{r}^{2}}{r^{3} \delta^{3}} .
$$

Rewrite (4.12) as

$$
k(\theta)=h(\theta-p(\theta))-p_{1}(\theta)+E_{1}(\theta)
$$

with

$$
E_{1}(\theta)=f_{1}(\theta,-1)+p_{1}(\theta)+h(\theta+f(\theta,-1))-h(\theta-p(\theta)) .
$$

Using the Taylor formula, we get

$$
h(\theta-p(\theta))=h(\theta)-\sum_{j=1}^{n} D_{j} h(\theta) \cdot p_{j}(\theta)+E_{2}(\theta)
$$

with

$$
E_{2}(\theta)=\sum_{i \leq j} p_{i}(\theta) p_{j}(\theta) D_{i} D_{j} h(\theta-t p(\theta))
$$

for some $t \in(0,1)$. Notice that $L_{1} h$ is a function in $\theta_{1}$ alone. Then we can write

$$
\sum_{j=1}^{n} D_{j} h(\theta) p_{j}(\theta)=D_{1} L_{1} h\left(\theta_{1}\right) p_{1}(\theta)+E_{3}(\theta)
$$

with

$$
E_{3}(\theta)=\sum_{j=2}^{n} \sum_{k=1}^{j} D_{k} L_{j} h(\theta) p_{k}(\theta) .
$$

Substituting (4.22) and (4.23) into (4.21), we get

$$
k=h-\left(D_{1} L_{1} h+1\right) p_{1}+E, \quad E=E_{1}+E_{2}-E_{3} .
$$

Using (4.9), we obtain

$$
k(\theta)=L_{0} h+L_{1} h\left(\theta_{1}\right)+E(\theta) .
$$

We now give some estimates of $E_{j}$. Using Cauchy inequalities, one gets

$$
\left\|D_{j} h\right\|_{(1-\delta) r} \leq B_{r}+(n-1) \frac{b_{r}}{r \delta}<1,
$$

in which (4.13) and (4.18) are used. One also has

$$
\left\|D_{i} D_{j} h\right\|_{(1-\delta) r} \leq \frac{1}{r \delta} .
$$


Fix $\theta \in S_{(1-3 \delta) r}$. From (4.2), we see that $\theta-p(\theta) \in S_{(1-2 \delta) r}$. Hence $|h(\theta+f(\theta,-1))-h(\theta-p(\theta))| \leq\|D h\|_{(1-2 \delta) r} \cdot\|f(\cdot,-1)+p(\cdot)\|_{(1-3 \theta) r}$. Together with (4.20) and (4.25), we get

$$
|h(\theta+f(\theta,-1))-h(\theta-p(\theta))| \leq \frac{c_{3} n b_{r}^{2}}{r^{3} \delta^{3}} .
$$

Hence

$$
\left\|E_{1}\right\|_{(1-3 \delta) r} \leq \frac{c_{3}(n+1) b_{r}^{2}}{r^{3} \delta^{3}} .
$$

Notice that $\theta-t p(\theta) \in S_{(1-2 \delta) r}$ for $|t| \leq 1$. From (4.14) and (4.26), it follows that

$$
\left\|E_{2}\right\|_{(1-3 \delta) r} \leq \frac{n^{2}}{r \delta}\left(\frac{c_{2} b_{r}}{r \delta}\right)^{2} .
$$

It is clear that for $j \geq 2,\left\|D_{k} L_{j} h\right\|_{(1-3 \delta) r} \leq b_{r} /(3 r \delta)$. Together with (4.14), we get

$$
\left\|E_{3}\right\|_{(1-3 \delta) r} \leq \frac{n(n-1) b_{r}}{3 r \delta} \cdot \frac{c_{2} b_{r}}{r \delta} .
$$

Let us put (4.27), (4.28) and (4.29) together in the form

$$
\left\|E_{1}\right\|_{(1-3 \delta) r}+\left\|E_{2}\right\|_{(1-3 \delta) r}+\left\|E_{3}\right\|_{(1-3 \delta) r} \equiv E_{0} \leq \frac{c_{4} b_{r}^{2}}{2 r^{3} \delta^{3}} .
$$

We now denote

$$
\begin{aligned}
\widetilde{B}_{(1-4 \delta) r} & =\max \left\{\left\|L_{0} k\right\|_{(1-4 \delta) r},\left\|D_{1} L_{1} k\right\|_{(1-4 \delta) r}\right\}, \\
\widetilde{b}_{(1-4 \delta) r} & =\max \left\{\left\|L_{2} k\right\|_{(1-4 \delta) r}, \ldots,\left\|L_{n} k\right\|_{(1-4 \delta) r}\right\} .
\end{aligned}
$$

Applying $D_{1} L_{1}$ to both sides of (4.24), we get

$$
\left\|D_{1} L_{1} k\right\|_{(1-4 \delta) r} \leq\left\|D_{1} L_{1} h\right\|_{(1-4 \delta) r}+\left\|D_{1} L_{1} E\right\|_{(1-4 \delta) r} .
$$

From (3.2) and Cauchy inequalities, we then obtain

$$
\left\|D_{1} L_{1} k\right\|_{(1-4 \delta) r} \leq B_{r}+\frac{2}{r \delta} E_{0} .
$$

It is easy to see that $L_{i} L_{j} \equiv 0$ for $i \neq j$. Hence, applying $L_{0}$ to both sides of (4.24) gives

$$
\left|L_{0} k\right| \leq B_{r}+E_{0} .
$$

We also have

$$
\left\|L_{j} k\right\|_{(1-3 \delta) r} \leq\left\|L_{j} E\right\|_{(1-3 \delta) r}, \quad j>1 .
$$

Hence

$$
\widetilde{b}_{(1-3 \delta) r} \leq 2 E_{0} .
$$


Substituting (4.30) into (4.31)-(4.33), we obtain the following.

Proposition 4.3. Assume that $0<r<1$ and $0<\delta<1 / 4$. Suppose that $h$ is a $2 \pi$-periodic holomorphic function satisfying (4.13) and (4.18). Let $\phi$ be defined by (4.11), and let $k$ be determined by (4.12) and (4.11). Then $\phi$ satisfies (4.19), and $k$ satisfies

$$
\widetilde{b}_{(1-4 \delta) r} \leq \frac{c_{4} b_{r}^{2}}{r^{3} \delta^{3}}
$$

and

$$
\widetilde{B}_{(1-4 \delta) r} \leq B_{r}+\frac{c_{4} b_{r}^{2}}{r^{4} \delta^{4}}
$$

4.3. Proof of Theorem 2.2. We put

$$
r_{m}=\frac{1}{2}\left(1+\frac{1}{m+1}\right) r, \quad m=0,1, \ldots
$$

Rewrite

$$
r_{m+1}=\left(1-4 \delta_{m}\right) r_{m}, \quad \delta_{m}=\frac{1}{4(m+2)^{2}}, \quad m=0,1, \ldots .
$$

Let us first prove a numerical result.

Lemma 4.4. Let $r_{m}, \delta_{m}$ be given as above. Assume that $b_{m}$ and $B_{m}(0 \leq$ $m \leq N)$ are non-negative numbers satisfying

$$
b_{m+1} \leq \frac{c_{4} b_{m}^{2}}{r_{m}^{3} \delta_{m}^{3}}
$$

and

$$
B_{m+1} \leq B_{m}+\frac{c_{4} b_{m}^{2}}{r_{m}^{4} \delta_{m}^{4}}
$$

for $0 \leq m<N$. Assume further that

$$
B_{0} \leq 1 / 4, \quad b_{0} \leq r_{0}^{3} \delta_{0}^{3} / c_{6}
$$

with $c_{6}=\max \left\{n c_{2}, 27 c_{4}\right\}$. Then for all $m>0$, we have

$$
b_{m} \leq r_{m}^{3} \delta_{m}^{3} / c_{6}, \quad B_{m} \leq 1 / 2 .
$$

Proof. We put

$$
\hat{b}_{m}=r_{m}^{3} \delta_{m}^{3} / c_{6}, \quad m \geq 0 .
$$

Assume that (4.39) holds for $m \leq m_{0}$. It is easy to see that

$$
\hat{b}_{m+1} / \hat{b}_{m}=\left(1-4 \delta_{m}\right)^{3} \frac{\delta_{m+1}^{3}}{\delta_{m}^{3}} \geq\left(1-4 \delta_{0}\right)^{3} \frac{\delta_{1}^{3}}{\delta_{0}^{3}}=1 / 27 \text {. }
$$


On the other hand, one has

$$
\frac{c_{4} b_{m_{0}}}{r_{m_{0}}^{3} \delta_{m_{0}}^{3}} \leq c_{4} / c_{6} \leq 1 / 27
$$

Hence

$$
b_{m_{0}+1} \leq b_{m_{0}} \frac{\hat{b}_{m_{0}+1}}{\hat{b}_{m_{0}}} \leq \hat{b}_{m_{0}+1} .
$$

As for the estimate of $B_{m_{0}+1}$, we have

$$
B_{m_{0}+1} \leq B_{0}+\sum_{j=0}^{m_{0}} \frac{c_{4} b_{j}^{3}}{r_{j}^{4} \delta_{j}^{4}} \leq 1 / 4+\sum_{j=0}^{m_{0}} r_{j}^{5} \delta_{j}^{5},
$$

in which the second inequality is obtained from the estimate of $b_{m}$ for $m \leq m_{0}+1$. Notice that $r_{0}<1$. Then it is easy to see that $B_{m_{0}+1} \leq 1 / 2$. Therefore, we have proved (4.39) by the induction.

For the proof of Theorem 2.2, we shall find a sequence of volumepreserving transformations $\phi_{m}$, and then define

$$
\theta_{1}+h^{(m+1)}(\theta)=\theta_{1}^{\prime}+h^{(m)}\left(\theta^{\prime}\right), \quad h^{(0)}=h
$$

with

$$
\phi_{m}: \theta^{\prime}=\theta+f^{(m)}(\theta,-1) .
$$

Denote

$$
\begin{gathered}
B_{m}=\max \left\{\left|L_{0} h^{(m)}\right|,\left\|D_{1} L_{1} h^{(m)}\right\|_{r_{m}}\right\}, \\
b_{m}=\max \left\{\left\|L_{2} h^{(m)}\right\|_{r_{m}}, \ldots,\left\|L_{n} h^{(m)}\right\|_{r_{m}}\right\} .
\end{gathered}
$$

The transformations $\phi_{m}$ will be constructed such that

$$
\left\|f^{(m)}(\cdot,-1)\right\|_{r_{m+1}} \leq r_{m} \delta_{m}
$$

In particular,

$$
\phi_{m}: S_{r_{m+1}} \rightarrow S_{r_{m}} .
$$

Furthermore, it required that (4.39) holds.

The existence of $\phi_{0}$ is based on the initial condition (2.6). In fact, we should choose $\epsilon$ such that (4.38) follows from (2.6). Hence, we get (4.13) and (4.18) for $h=h^{(0)}$. Let $\phi_{0}=\phi$ be as in Proposition 4.3. Then (4.19) implies that (4.41) holds for $m=0$. Inductively, we assume that $\phi_{0}, \ldots, \phi_{m_{0}}$ are constructed such that (4.41) and (4.39) are satisfied for $m \leq m_{0}$. Then Proposition 4.3 implies that there exists a transformation $\phi^{(m+1)}$ satisfies (4.41). The existence of $\phi_{0}, \ldots, \phi_{m_{0}+1}$ also implies that (4.34) and (4.35) hold for $m \leq m_{0}+1$. Finally, from Lemma 4.4, we obtain (4.39) for $m=m_{0}+1$. By the induction, we 
may construct a sequence of transformations $\phi_{m}$ satisfying (4.41) such that the corresponding $h^{(m)}$ satisfies (4.39).

We now want to prove that $\phi_{0} \circ \ldots \circ \phi_{m}$ converges on $S_{r_{0} / 2}$ as $m \rightarrow \infty$. We first notice that $S_{r_{0} / 2} \subset S_{r_{m}}$ for all $m$. From (4.42), it follows that

$$
\begin{aligned}
\left\|\phi_{0} \circ \ldots \circ \phi_{m}-\mathrm{Id}\right\|_{r_{0} / 2} & \leq \sum_{j=0}^{m}\left\|\phi_{j} \circ \ldots \circ \phi_{m}-\phi_{j+1} \circ \ldots \circ \phi_{m}\right\|_{r_{0} / 2} \\
& \leq \sum_{j=0}^{m}\left\|f^{(j)}\left(\phi_{j+1} \circ \ldots \circ \phi_{m},-1\right)\right\|_{r_{0} / 2} .
\end{aligned}
$$

On the other hand, from (4.41), we get

$$
\left\|f^{(m)}(\cdot,-1)\right\|_{r_{m+1}} \leq \delta_{m}
$$

Therefore, $\phi_{0} \circ \ldots \circ \phi_{m}$ converges to a holomorphic mapping $\Phi_{\infty}: S_{r_{0} / 2} \rightarrow$ $\mathbb{C}^{n}$. It is clear that $\Phi_{\infty}-\mathrm{Id}: \mathbb{R}^{n} \rightarrow \mathbb{R}^{n}$ is $2 \pi$-periodic. Hence, the restriction of $\Phi_{\infty}$ to $\mathbb{R}^{n}$ generates a volume-preserving diffeomorphism $\Phi$ of $T^{n}$ satisfying (2.7), where

$$
k=\lim _{m \rightarrow \infty}\left\{L_{0} h^{(m)}+L_{1} h^{(m)}\right\} .
$$

One can further achieve $[k]=L_{0} k=0$ by applying a suitable translation (3.25). Therefore, the proof of Theorem 2.2 is complete.

\section{The ReAlization OF COMPLEX $n$-FORMS}

In this section, we shall give a proof for Theorem 1.4. As in section 4 , we shall first consider an approximation to the nonlinear equation. We then use the KAM method to show the existence of a convergent solution to the original equation.

5.1. A linearized equation. For the proof of Theorem 1.4, we try to find a time-1 mapping $\varphi$ of a holomorphic vector field in $A_{r}$ given by

$$
v(z)=\sum_{j=1}^{n} q_{j}(z) \frac{\partial}{\partial z_{j}}
$$

such that $\varphi^{*} \Omega=\omega$, i. e.

$$
\operatorname{det} D \varphi=1+a(z) \text {. }
$$

Notice the following determinant formula (see [14], p. 142)

$$
\log \operatorname{det} D \varphi_{t}=\int_{0}^{t} \sigma \circ \varphi_{s}(z) d s, \quad-1 \leq t \leq 1
$$


with

$$
\sigma(z)=\sum_{j=1}^{n} \partial_{j} q_{j}, \quad \partial_{j} \equiv \frac{\partial}{\partial z_{j}}
$$

Thus, we are led to the following functional equation

$$
\int_{0}^{1} \sigma \circ \varphi_{t}(z) d t=\log (1+a(z)) .
$$

Instead of solving (5.3), we now consider its linearized equation

$$
\sum_{j=1}^{n} \partial_{j} q_{j}=a(z)
$$

To solve the linearized equation, we decompose

$$
a(z)=K_{1} a(z)+\ldots+K_{n} a(z)+K_{n+1} a(z)
$$

with

$$
K_{j} a(z)=\frac{1}{z_{1} \ldots z_{j-1}} \sum_{i_{j} \neq-1} a_{-1, \ldots,-1, i_{j}, \ldots, i_{n}} z_{j}^{i_{j}} \ldots z_{n}^{i_{n}}
$$

for $1 \leq j \leq n$. It is easy to see that the condition (1.5) holds for $\omega$, if and only if

$$
K_{n+1} a(z)=\frac{a_{-1, \ldots,-1}}{z_{1} \ldots z_{n}} \equiv 0
$$

We also have

$$
\sum_{l=j}^{n+1} K_{l} a(z)=\frac{z_{1}^{-1} \ldots z_{j-1}^{-1}}{(2 \pi i)^{j-1}} \int_{\left|z_{1}\right|=\ldots=\left|z_{j-1}\right|=1} a(z) d z_{1} \wedge \ldots \wedge z_{j-1} .
$$

Notice that $\left|z_{j}\right|<e$ for $z \in A_{r}, 0<r<1$. Hence

$$
\left\|K_{j} a\right\|_{r} \leq 2 e^{j}\|a\|_{r}
$$

We now let $q_{j}(z)$ be the unique Laurent series, containing no terms of the form $z_{1}^{i_{1}} \ldots z_{n}^{i_{n}}$ with $i_{j}=0$, such that $\partial_{j} q_{j}(z)=K_{j} a(z)$. Then (5.5) implies that $q_{1}, \ldots, q_{n}$ satisfy (5.4). It is easy to see that

$$
q_{j}(z)=i D_{j}^{-1}\left(z_{j} K_{j} a(z)\right), \quad z=\left(\left|z_{1}\right| e^{i \theta_{1}}, \ldots,\left|z_{n}\right| e^{i \theta_{n}}\right) .
$$

Then, it follows from (3.5) and (5.6) that

$$
\left\|q_{j}\right\|_{r} \leq 4 \pi e^{j+1}\|a\|_{r}, \quad 0<r<1 .
$$

Let $v$ be the vector field defined by (5.1) and $\varphi_{t}$ the flow of $v$. Put

$$
\varphi_{t}: z_{j}^{\prime}=z_{j} g_{j}(z, t), \quad g_{j}(z, 0) \equiv 1 .
$$

We have the following. 
Lemma 5.1. Let $a(z), \varphi_{t}, g_{j}(z, t)$ be as above. Assume that

$$
\|a\|_{r} \leq \frac{r \delta^{2}}{4 e^{n+2} \pi}
$$

Then for $0<r<1,0<\delta<1 / 2$ and $|t| \leq 1$, we have

$$
\left\|\log g_{j}(\cdot, t)\right\|_{(1-\delta) r} \leq r \delta^{2} .
$$

Proof. We consider the vector field

$$
\widetilde{v}(\theta)=\sum_{j=1}^{n} p_{j}(\theta) \frac{\partial}{\partial \theta_{j}}, \quad p_{j}(\theta)=-i e^{-i \theta_{j}} q_{j}(\pi(\theta)) .
$$

Clearly, $\widetilde{v}$ is a holomorphic vector field in $S_{r}$ with $\pi_{*} \widetilde{v}=v$. Let $\phi_{t}$ be the flow of $\widetilde{v}$. Then $\varphi_{t}=\pi \phi_{t} \pi^{-1}$. Hence

$$
g_{j}(z, t)=e^{i f_{j}\left(\log z_{1}, \ldots, \log z_{n}, t\right)} .
$$

Since $0<r<1$, we have $\left\|p_{j}\right\|_{r} \leq e\left\|q_{j}\right\|_{r}$. From (5.8) and (5.9), it is easy to see that

$$
\left\|p_{j}\right\|_{r} \leq r \delta^{2} .
$$

In particular, $p$ satisfies (4.2) for $r_{1}=r$. Now (5.10) is obtained from (4.3), (5.11) and (5.12) immediately. This completes the proof of Lemma 5.1 .

Let $\varphi_{t}$ be the flow given in Lemma 5.1. We put $\psi=\varphi_{-1}$ and denote

$$
\psi^{*} \omega=\hat{\omega}, \quad \hat{\omega}(z)=(1+\hat{a}(z)) d z_{1} \wedge \ldots \wedge d z_{n} .
$$

Then

$$
1+\hat{a}(z)=(1+a \circ \psi) \operatorname{det} D \psi
$$

We are ready to prove the following.

Proposition 5.2. Let $a(z)$ and $\varphi_{t}$ be given as in Lemma 5.1. Let $\hat{a}(z)$ be defined as above. Then

$$
\varphi_{t}: A_{(1-2 \delta) r} \rightarrow A_{(1-\delta) r}
$$

and

$$
\|\hat{a}\|_{(1-2 \delta) r} \leq \frac{c_{7}\|a\|_{r}^{2}}{r \delta}
$$

for some constant $c_{7}$ depending only on $n$.

Proof. From (5.10), it follows that (5.14) holds. Let

$$
Q=\log (1+a \circ \psi)+\log \operatorname{det} D \psi .
$$


From (5.2), we get

$$
Q=\log (1+a \circ \psi)-a \circ \psi+\int_{0}^{-1}\left(a \circ \varphi_{s}-a \circ \psi\right) d s .
$$

By Cauchy inequalities, we have

$$
\left\|\partial_{j} a\right\|_{(1-\delta) r} \leq \frac{e\|a\|_{r}}{r \delta}
$$

Notice that

$$
a\left(\varphi_{t}(z)\right)-a(\psi(z))=\sum_{j=1}^{n} \int_{-1}^{t} \partial_{j} a\left(\varphi_{s}(z)\right) q_{j}\left(\varphi_{s}(z)\right) d s .
$$

Hence, (5.14) leads to

$$
\left\|a \circ \varphi_{t}-a \circ \psi\right\|_{(1-2 \delta) r} \leq \frac{n e}{r \delta}\|a\|_{r}\|q\|_{r} .
$$

From (5.8), we then obtain

$$
\left\|\int_{0}^{-1}\left(a \circ \varphi_{s}-a \circ \psi\right) d s\right\|_{(1-2 \delta) r} \leq \frac{4 n \pi e^{n+2}}{r \delta}\|a\|_{r}^{2} .
$$

Notice that $\|a\|_{r}<1 / 2$. Hence, (3.14) gives us

$$
\|\log (1+a \circ \psi)-a \circ \psi\|_{(1-2 \delta) r} \leq 2\|a\|_{r}^{2} .
$$

From (5.16), we now see that

$$
\|Q\|_{(1-2 \delta) r} \leq \frac{4 n \pi e^{n+2}+1}{r \delta}\|a\|_{r}^{2} .
$$

In particular, (5.9) and (3.15) imply that

$$
\|\hat{a}\|_{(1-2 \delta)}=\left\|e^{Q}-1\right\|_{(1-2 \delta)} \leq e\|Q\|_{(1-2 \delta)} .
$$

Together with (5.17), we obtain (5.15).

5.2. Proof of Theorem 1.4. The rest of the proof of Theorem 1.4 will be given along the lines of the proof of Theorem 2.2. We put

$$
r_{m+1}=\left(1-2 \delta_{m}\right) r_{m}, \quad \delta_{m}=\frac{e^{-2} n^{-1}}{2(m+2)^{2}}, \quad m=0,1, \ldots
$$

The following numerical result can be proved as Lemma 4.4.

Lemma 5.3. Let $r_{m}, \delta_{m}$ be given as above. Assume that $a_{0}, a_{1}, \ldots, a_{N}$ are non-negative numbers satisfying

$$
a_{m+1} \leq \frac{c_{7} a_{m}^{2}}{r_{m} \delta_{m}}, \quad 0 \leq m<N
$$


and

$$
a_{0} \leq r_{0} \delta_{0}^{2} / c_{8}
$$

for $c_{8}=\max \left\{c_{7}, 4 e^{n+2} \pi\right\}$, then

$$
a_{m} \leq r_{m} \delta_{m}^{2} / c_{8}, \quad m \geq 0 .
$$

For the proof of Theorem 1.4, we shall find a sequence of transformations

and then put

$$
\psi_{m}: z_{j}^{\prime}=z_{j} g_{j}^{(m)}(z), \quad j=1, \ldots, n
$$

$$
\left(1+a^{(m+1)}(z)\right) \Omega=\psi_{m}^{*}\left(\left(1+a^{(m)}(z)\right) \Omega\right) .
$$

Denote

$$
a_{m}=\left\|a^{(m)}\right\|_{r_{m}} .
$$

We require that (5.20) holds and $\psi_{m}$ satisfies

$$
\left\|\log g_{j}^{(m)}\right\|_{r_{m+1}} \leq r_{m} \delta_{m}^{2}
$$

In particular, (5.22) implies that

$$
\psi_{m}: A_{r_{m+1}} \rightarrow A_{r_{m}} .
$$

To determine $\psi_{m}$, we now let $a^{(0)}(z)=a(z)$ be as in Theorem 1.4 . Then for $\epsilon$ small, we know that $a_{0}$ satisfies (5.19). By applying Lemma 5.1 to $a=a^{(0)}$ and $r=r_{0}$, we find a transformation $\psi_{0}=\varphi_{-1}$ as in Lemma 5.1. Next, we define $a^{(1)}(z)$ by (5.21). From Proposition 5.2, we know that $a_{0}$ and $a_{1}$ satisfies (5.18). Hence, Lemma 5.3 implies that (5.20) holds for $a_{1}$. In return, we construct a transformation $\psi_{1}=\psi$ as in Lemma 5.1 for $a=a^{(1)}$ and $r=r_{1}$, so $a^{(2)}(z)$ is determined by (5.21). Through this recursive process, we can construct a sequence of transformations $\psi_{m}$ such that (5.20)-(5.23) hold.

Notice that $r_{m}>r_{0} / 2$ for all $m$. Then (5.23) implies that $\psi_{0} \circ \ldots \circ \psi_{m}$ is well-defined on the domain $A_{r_{0} / 2}$. We have

$$
\left\|\psi_{k}-\mathrm{Id}\right\|_{r_{k+1}} \leq e \max _{1 \leq j \leq n}\left\{\left\|g_{j}^{(k)}-1\right\|_{r_{k+1}}\right\} \leq e^{2} r_{k} \delta_{k}^{2},
$$

where the last inequality comes from (5.22) and (3.15). Hence, (5.23) implies that

$$
\left\|\psi_{k} \circ \ldots \circ \psi_{m}-\psi_{k+1} \circ \ldots \circ \psi_{m}\right\|_{r_{0} / 2} \leq\left\|\psi_{k}-\operatorname{Id}\right\|_{r_{k+1}} \leq e^{2} r_{0} \delta_{k}^{2} .
$$

Thus, the sequence $\psi_{0} \circ \ldots \circ \psi_{m}$ converges on $A_{r_{0} / 2}$, of which the limit mapping $\psi$ satisfies

$$
\|\psi-\mathrm{Id}\|_{r_{0} / 2} \leq e^{2} r_{0} \sum_{m=0}^{\infty} \delta_{m}^{2} \leq \frac{r_{0}}{16 n^{2} e^{2}}
$$


where the last inequality is obtained from the choice of $\delta_{m}$ and the elementary inequality $(m+2)^{4} \geq 4(m+1)(m+2)$.

It is clear that $\psi^{*} \omega=\Omega$ on $A_{r_{0} / 2}$. We now can complete the proof of Theorem 1.4 by showing that $\psi$ has an inverse mapping $\varphi: A_{r_{0} / 8} \rightarrow$ $A_{r_{0} / 4}$. To this end, we fix $z \in A_{r_{0} / 8}$ and consider the mapping

$$
T: \xi \rightarrow z-\psi(\xi)+\xi \text {. }
$$

From (5.24), it is clear that $T: A_{r_{0} / 4} \rightarrow A_{r_{0} / 4}$. Next, we want to show that $T$ is a contraction mapping. For if $\xi^{\prime}, \xi^{\prime \prime}$ are two distinct points in $A_{r_{0} / 4}$, we choose $\theta^{\prime}, \theta^{\prime \prime} \in S_{r_{0} / 4}$ such that $\pi\left(\theta^{\prime}\right)=\xi^{\prime}$ and $\pi\left(\theta^{\prime \prime}\right)=\xi^{\prime \prime}$. We may assume that

$$
\left|\operatorname{Re}\left(\theta_{j}^{\prime \prime}-\theta_{j}^{\prime}\right)\right| \leq \pi, \quad j=1, \ldots, n .
$$

Let $\gamma(t)=\pi\left((1-t) \theta^{\prime}+t \theta^{\prime \prime}\right)$. Then $\gamma:[0,1] \rightarrow A_{r_{0} / 4}$, and

$$
\left\|\gamma^{\prime}(t)\right\| \leq e\left\|\theta^{\prime \prime}-\theta^{\prime}\right\|, \quad t \in[0,1] .
$$

Using Cauchy inequalities, we also obtain from (5.24) that

$$
\|D \psi-\mathrm{Id}\|_{r_{0} / 4} \leq \frac{1}{4 e n}
$$

Combining with the formula

$$
T\left(\xi^{\prime \prime}\right)-T\left(\xi^{\prime}\right)=-\int_{0}^{1}(D \psi-\mathrm{Id}) \gamma^{\prime}(t) d t
$$

we obtain

$$
\left\|T\left(\xi^{\prime \prime}\right)-T\left(\xi^{\prime}\right)\right\| \leq\left\|\theta^{\prime \prime}-\theta^{\prime}\right\| / 4 .
$$

Since $\left|\xi_{j}^{\prime}\right|,\left|\xi_{j}^{\prime \prime}\right| \geq r_{0} / 4$, then we have

$$
\left|\xi_{j}^{\prime \prime}-\xi_{j}^{\prime}\right| \geq|| \xi_{j}^{\prime \prime}|-| \xi_{j}^{\prime}|| \geq e^{-r_{0} / 4}\left|\operatorname{Im}\left(\theta_{j}^{\prime \prime}-\theta_{j}^{\prime}\right)\right|
$$

and

$$
\left|\xi_{j}^{\prime \prime}-\xi_{j}^{\prime}\right| \geq e^{-r_{0} / 4}\left|\xi_{j}^{\prime \prime} /\right| \xi_{j}^{\prime \prime}\left|-\xi_{j}^{\prime} /\right| \xi_{j}^{\prime}|| \geq 2 e^{-r_{0} / 4}\left|\operatorname{Re}\left(\theta_{j}^{\prime \prime}-\theta_{j}^{\prime}\right)\right| / \pi .
$$

Thus, we get

$$
\left\|\xi^{\prime \prime}-\xi^{\prime}\right\| \geq \sqrt{2} e^{-1 / 4}\left\|\theta^{\prime \prime}-\theta^{\prime}\right\| / \pi .
$$

Combining with (5.26), we obtain

$$
\left\|T\left(\xi^{\prime \prime}\right)-T\left(\xi^{\prime}\right)\right\| \leq \frac{\pi e^{1 / 4}}{4 \sqrt{2}}\left\|\xi^{\prime \prime}-\xi^{\prime}\right\|, \quad \xi^{\prime}, \xi^{\prime \prime} \in A_{r_{0} / 4} .
$$

This shows that $T: A_{r_{0} / 4} \rightarrow A_{r_{0} / 4}$ is a contraction mapping. Therefore, the fixed point theorem implies that $\psi$ has a unique inverse $\varphi: A_{r_{0} / 8} \rightarrow$ $A_{r_{0} / 4}$. From (5.25), it is also clear that $\varphi$ is holomorphic. The proof of Theorem 1.4 is complete. 
Acknowledgment. The author would like to thank Professor Sidney Webster for the constant encouragement and helpful discussions.

\section{REFERENCES}

[1] P. Ahern and W. Rudin, Totally real embeddings of $S^{3}$ in $\mathbb{C}^{3}$, Proc. Amer. Math. Soc., 94(1983), no.3, 360-462.

[2] E. Bishop, Differentiable manifolds in complex Euclidean space, Duke Math. J., 32(1965), 1-22.

[3] C. Ehresmann, Sur les espaces fibrés différentiables, C. R. Acad. Sci. Paris, 224(1947), 1611-1612.

[4] F. T. Farrell, The obstruction to fibering a manifold over a circle, Indiana Univ. Math. J., 21(1971), no.4, 315-346.

[5] F. Forstnerič, Complex tangents of real surfaces in complex surfaces, Duke Math. J., no.2, 67(1992), 353-376.

[6] X. Gong, Real analytic submanifolds under unimodular transformations, Proc. Amer. Math. Soc., 123(1995), no.1, 191-200.

[7] V. Guillemin and A. Pollack, Differential Topology, Prentice-Hall, Englewood Cliffs, New Jersey, 1974.

[8] M. W. Hirsch, Differential Topology, Graduate Text in Math. 33, SpringerVerlag, New York, 1976.

[9] S. T. Hu, Homotopy Theory, Academic Press, New York and London, 1959.

[10] H. F. Lai, Characteristic classes of real manifolds immersed in complex manifolds, Trans. A.M.S., 170(1972), 1-33.

[11] J. K. Moser, On the volume elements on a manifold, Trans. Amer. Math. Soc., 120(1965), 286-294.

[12] G. de Rham, Variétés différentiables, Hermann, Paris, 1955.

[13] W. Rudin, Totally real Klein Bottles in $\mathbb{C}^{2}$, Proc. Amer. Math. Soc., 82(1981), 653-654.

[14] C. L. Siegel and J. K. Moser, Lectures on Celestial Mechanics, Springer-Verlag, New York, 1971.

[15] N. Steenrod, The Topology of Fibre Bundles, Princeton University Press, Princeton, New Jersey, 1951.

[16] D. Tischler, On fibering certain foliated manifold over $S^{1}$, Topology, 9(1970), 153-154.

[17] S. M. Webster, Minimal surfaces in a Kähler surface, J. Diff. Geom., 20(1984), 463-470.

[18] S. M. Webster, The Euler and Pontrjagin numbers of an n-manifold in $\mathbb{C}^{n}$, Comment. Math. Helv., 60(1985), no.2, 193-216.

[19] A. Weinstein, Lectures on Symplectic Geometry, Reg. Conf. Ser. Math. 29, Amer. Math. Soc., Providence, 1977.

[20] R. O. Wells, Compact real submanifolds of a complex manifold with nondegenerate holomorphic tangent bundles, Math. Ann., 179(1969), 123-129.

[21] H. Whitney, On regular closed curves in the plane, Compositio Math., 4(1937), 276-284.

Institute for Advanced Study, School of Mathematics, Princeton, NJ 08540

E-mail address: gong@math.ias.edu 